\title{
The Bers-Greenberg Theorem and the Maskit Embedding for Teichmüller spaces
}

\author{
Pablo Arés Gastesi* \\ August 2, 2018 \\ To the memory of Lipman Bers
}

\begin{abstract}
The Bers-Greenberg theorem tells that the Teichmüller space of a Riemann surface with branch points (orbifold) depends only on the genus and the number of special points, but not on the particular ramification values. On the other hand, the Maskit embedding provides a mapping from the Teichmüller space of an orbifold, into the product of one dimensional Teichmüller spaces. In this paper we prove that there is a set of isomorphisms between one dimensional Teichmüller spaces that, when restricted to the image of the Teichmüller space of an orbifold under the Maskit embedding, provides the Bers-Greenberg isomorphism.
\end{abstract}

\section{Introduction.}

The purpose of this paper is to connect two important results in Teichmüller theory, namely the Bers-Greenberg theorem and the Maskit embedding. We will prove that the isomorphism given by the former theorem splits into the product of one dimensional isomorphisms in the Maskit embedding.

*Partially supported by SUNY at Stony Brook and Tata Institute. 1991 Mathematics Subject Classification. Primary 32G15, 30F40; Secondary 30F10, 32 G05 
Let $S$ be an orbifold with hyperbolic signature $\left(p, n ; \nu_{1}, \ldots, \nu_{n}\right)$. A key result of $\mathrm{B}$. Maskit tells that $S$ can be written as the quotient $\Delta / \Gamma$, where $\Gamma$ is a terminal regular b-group (it uniformizes $S$ and a finite number of orbifolds of type $(0,3)$, which carry no moduli) and $\Delta$ is a simply connected $\Gamma$-invariant open subset of the regular set $\Omega(\Gamma)$ of $\Gamma$. The Bers-Greenberg theorem says that the complex structure of the Teichmüller space of $\Gamma, T(\Gamma)$, depends only on $(p, n)$, and not on the particular values $\nu_{j}$.

Applying the combination theorems (these are just tools to construct Kleinian groups from smaller groups), Maskit produced an embedding of the Teichmüller space $T(\Gamma)$ into the product $\prod_{j=1}^{3 p-3+n} T\left(\Gamma^{j}\right)$, where the $\Gamma^{j}$ 's are certain subgroups of $\Gamma$, whose Teichmüller spaces have dimension 1. One can ask whether it is possible to put the Bers-Greenberg theorem and the Maskit embedding together in a single result. More precisely, we have a diagram

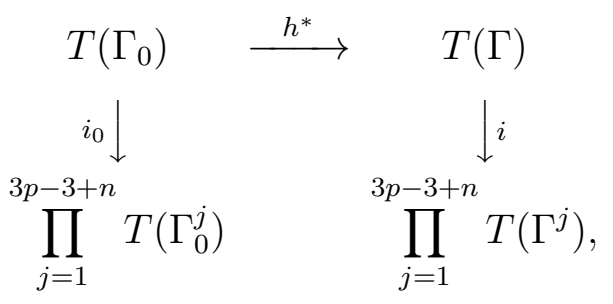

where $\Gamma_{0}$ and $\Gamma$ are terminal regular b-groups of the same type $(p, n), i_{0}$ and $i$ are the Maskit embeddings, and $h^{*}$ is an isomorphism obtained in the proof of the Bers-Greenberg theorem. The main result of this paper is that the above diagram can be closed in a commutative way with a set of one dimensional isomorphisms.

Theorem 1 Let $S$ be an orbifold with hyperbolic signature. Let $S_{0}$ be the orbifold obtained by removing from $S$ all points with finite ramification value. Let $\mathcal{P}$ be a maximal partition on $S$ (and $S_{0}$ ). Assume $\Gamma$ and $\Gamma_{0}$ are terminal regular b-groups uniformizing $(S, \mathcal{P})$ and $\left(S_{0}, \mathcal{P}\right)$ on the invariant components $\Delta$ and $\Delta_{0}$ respectively. Then there exist a choice of modular subgroups $\Gamma^{1}, \ldots, \Gamma^{3 p-3+n}$ of $\Gamma$, and $\Gamma_{0}^{1}, \ldots, \Gamma_{0}^{3 p-3+n}$ of $\Gamma_{0}$, and a set of isomorphisms $h_{j}^{*}: T\left(\Gamma_{0}^{j}\right) \rightarrow T\left(\Gamma^{j}\right), 1 \leq j \leq 3 p-3+n$, such that the restriction of $\left(h_{1}^{*}, \ldots, h_{3 p-3+n}^{*}\right)$ to $i_{0}\left(T\left(\Gamma_{0}\right)\right)$ closes the above diagram in a commutative way. 
Remark. Observe that we can choose $i^{-1} \circ h^{*} \circ i_{0}$ as an isomorphism between $i_{0}\left(T\left(\Gamma_{0}\right)\right)$ and $i(T(\Gamma))$, but our result is deeper since it produces isomorphisms at the one dimensional level that are "compatible" with the Maskit embedding.

This paper is organized as follows. In $\S 2$ we give the necessary background on Kleinian groups and Teichmüller spaces; in $\S 3$ we prove the BersGreenberg theorem adjusted to our case following [5]; $\S 4$ contains some technical results; finally in $\S 5$ we prove theorem 1 .

Acknowledgements. I would like to thank my advisor, Irwin Kra, for posing the question that made possible this paper and for all his help. Special thanks are due to M. Lyvbich, who provided the proof of lemma 4.3., and C.Zhang for many useful conversations. I am also very grateful to the referee for many comments that helped to clarify this paper. Thanks are also due to K. P. Sivaraman the typing the manuscript.

\section{Background on Kleinian groups and Te- ichmüller spaces.}

2.1. Let $\bar{S}$ be compact Riemann surface of genus $p$, and let $x_{1}, \ldots, x_{n}$ be $n \geq 0$ distinct points of $\bar{S}$. Let $\nu_{1}, \cdots, \nu_{n} \in Z^{+} \cup\{\infty\}, \nu_{j} \geq 2$, satisfying $2 p-2+n-\sum_{j=1}^{n} \frac{1}{\nu_{j}}>0$. The tuple $\sigma=\left(p, n ; \nu_{1}, \ldots, \nu_{n}\right)$ is called a hyperbolic signature, and $(p, n)$ is called the type of $\sigma$; the $\nu_{j}$ 's are called ramification values. Assign the value $\nu_{j}$ to $x_{j}$. An orbifold structure on $\bar{S}$ is given by a branched covering from the upper half plane $H$ onto $S=\bar{S}-\left\{x_{j} ; v_{j}=\infty\right\}$, such that the natural projection $H \stackrel{\pi}{\rightarrow} S$ is $\nu_{j}$-to- 1 in a neighbourhood of $x_{j}$ whenever $\nu_{j}<\infty$ (by an abuse of notation we will say that $S$ is an orbifold with signature $\sigma$ and of type $(p, n))$. It is a consequence of the PoincaréKoebe uniformization theorem that there exists a Fuchsian group $F$, such that $H / F \cong S$, but we are interested on a different representation of $S$.

A maximal partition, $\mathcal{P}=\left\{a_{1}, \ldots, a_{3 p-3+n}\right\}$, on $S$ is a set of simple closed disjoint unoriented curves on $S_{0}=S-\left\{x_{j} ; \nu_{j}<\infty\right\}$, such that no curve of $\mathcal{P}$ bounds a disc or a punctured disc on $S_{o}$, and no two curves of $\mathcal{P}$ bound a cylinder on $S_{0}$.

For a Kleinian group $G$, let $\Omega(G)$ denote the non-empty open set of points of $\hat{\mathbf{C}}=C \cup\{\infty\}$ where $G$ acts discontinuosuly. The components of $\Omega(G)$ are 
called the components of $G$.

Theorem 2 (Maskit uniformization) Given an orbifold $S$ with hyperbolic signature $\sigma$ and maximal partition $\mathcal{P}$, there exists a (unique up to conjugation by a Möbius transformation) geometrically finite Kleinian group $\Gamma$, called a terminal regular b-group, with a simply connected invariant component $\Delta$, such that:

1.- $\Delta / \Gamma$ is conformally equivalent to $S$;

2.- the curves of $\mathcal{P}$ are in one-to-one correspondence with the conjugacy classes of maximal cyclic subgroups of $\Gamma$ generated by accidental parabolic elements;

3.- $(\Omega(\Gamma)-\Delta) / \Gamma$ is the (finite) union of the orbifolds of type $(0,3)$ obtained by squeezing each curve of $\mathcal{P}$ to a point, and discarding all orbifolds of signature $(0,3,2,2, \infty)$ that appear.

See Maskit's book [12] for the definition of geometrically finite and accidental parabolic elements; they are technical terms not needed to understand the proofs of this paper.

2.2. Let $G$ be a finitely generated non-elementary Kleinian group, and let $A$ be an open $G$-invariant subset of $\Omega(G)$. Let $L^{\infty}(G, A)$ denote the set of essentially bounded (classes of) functions $\mu$, supported on $A$ and such that $(\mu \circ \gamma) \overline{\gamma^{\prime}} / \gamma^{\prime}=\mu$, for all $\gamma \in G$. Let $M(G, A)$ be the unit ball of $L^{\infty}(G, A)$; the classical theory of quasiconformal mappings tells us that if $w$ is a quasiconformal homeomorphism of $\hat{\mathbf{C}}$ satisfying the equation $(*) w_{\bar{z}}=$ $\mu w_{z}$, for some $\mu \in M(G, A)$, then the group $w \Gamma w^{-1}$ is again a group of Möbius transformations (see [13]). Two functions in $M(G, A), \mu_{1}$ and $\mu_{2}$, are equivalent if $w^{\mu_{1}} \gamma\left(w^{\mu_{1}}\right)^{-1}=w^{\mu_{2}} \gamma\left(w^{\mu_{2}}\right)^{-1}$ for all $\gamma \in G$, where $w^{\mu}$ is the unique solution of $(*$ ) fixing $\infty, 0$ and 1 (as a technical assumption, we need to suppose that these three points do not lie in $\Omega(G)$; this can always be achieved by conjugating by a Möbius transformation since $G$ is non-elementary). The set of equivalence classes of elements of $M(G, A)$ is called the Teichmüller space of $G$ supported on $A$, and it is denoted by $T(G, A)$. This set has a unique complex structure so that the natural projection from $M(G, A)$ onto $T(G, A)$ is holomorphic. In the case we are interested on we will take $G=\Gamma$ as in the Maskit uniformization theorem, and $A=\Delta$; we then have $T(\Gamma, \Delta)=T(\Gamma, \Omega(\Gamma)$ ) (since the orbifolds uniformized by $\Gamma$, other than $S$, carry no moduli). We will denote this set by $T(\Gamma)$. It is a well known fact 
that $T(\Gamma)$ is a complex manifold of dimension $3 p-3+n$. One can ask up to what point the complex structure of this space depends on the values $\nu_{j}$ appearing in the signature $\sigma$ of $S \cong \Delta / \Gamma$. The following theorem answers that question.

Theorem 3 (Bers-Greenberg [4]) Assume $\Gamma_{1}$ and $\Gamma_{2}$ are two terminal regular b-groups with invariant components $\Delta_{1}$ and $\Delta_{2}$ such that the orbifolds $\Delta_{1} / \Gamma_{1}$ and $\Delta_{2} / \Gamma_{2}$ are of the same type $(p, n)$. Then the Teichmüller spaces $T\left(\Gamma_{1}\right)$ and $T\left(\Gamma_{2}\right)$ are biholomorphically equivalent.

Remark. Although the theorem was initially proven for Fuchsian groups, the result is also valid in the case of terminal regular b-groups; we will provide a proof of it in $\S 3$.

2.3. The Maskit uniformization theorem produces an embedding of $T(\Gamma)$ into the product of one-dimensional Teichmüller spaces as follows. Let $T_{j}$, $1 \leq j \leq 3 p-3+n$, be the connected component of $S-\left\{a_{k} ; a_{k} \in C, k \neq j\right\}$ containing $a_{j}$ (these sets are called the modular parts of $S$ ). Let $D_{j}$ be a component of $\pi^{-1}\left(T_{j}\right)$, where $\pi: \Delta \rightarrow S$ is the natural projection, and let $\Gamma^{j}=\left\{\gamma \in \Gamma ; \gamma\left(D_{j}\right)=D_{j}\right\}$. The groups $\Gamma^{j}$ are known as modular subgroups of $\Gamma$; observe that if $\tilde{D}_{j}$ is another component of $\pi^{-1}\left(T_{j}\right)$ and $\tilde{\Gamma}^{j}$ is the corresponding subgroup, then $\Gamma^{j}$ and $\tilde{\Gamma}^{j}$ are conjugated on $\Gamma$. From the work of Maskit one can see that $\Gamma^{j}$ is a terminal regular b-group of type $(0,4)$ or $(1,1)$ with invariant component $\Delta^{j} \supset \Delta$. It is clear that $M\left(\Gamma^{j}, \Delta^{j}\right)$ contains $M(\Gamma, \Delta)$, but we have a stronger result.

Theorem 4 (Maskit embedding; Maskit [11], Kra [8]) The mapping given by restriction $T(\Gamma) \rightarrow \prod_{j=1}^{3 p-3+n} T\left(\Gamma^{j}\right)$ is holomorphic and injective with open image.

By a result of Gentilesco [6], the image of $T(\Gamma)$ is not the whole set $\prod_{j=1}^{3 p-3+n} T\left(\Gamma^{j}\right)$, unless we are in the trivial case of $\operatorname{dim} T(\Gamma)=1$.

2.4. We need to define one more set of holomorphic functions related to Kleinian groups. Given $\Gamma$ and $\Delta$ as above (theorem 2), $Q(\Gamma, \Delta)$ will denote the space of quadratic differentials (for $\Gamma$ on $\Delta$ ) consisting on the functions 
$f$, holomorphic on $\Delta$ such that $(f \circ \gamma)\left(\gamma^{\prime}\right)^{2}=f$ for all $\gamma \in \Gamma$, and with finite norm

$$
\|f\|=\frac{1}{2} \iint_{\Delta / \Gamma}|f(z) d z \wedge d \bar{z}|<\infty .
$$

The elements of $M(\Gamma, \Delta)$ of the form $k \frac{\bar{\varphi}}{\varphi \varphi}$, for some $\varphi \in Q(\Gamma, \Delta)$ and some $k \in(0,1)$ real, are called Teichmüller differentials. Teichmüller's theorem (Ahlfors [罒, Bers [3]) tells that on each class of $T(\Gamma)$ there exists a unique Teichmüller differential.

2.5. Finally we define the Teichmüller space of an orbifold and study the relationship with the Teichmüller space of Kleinian groups. Let $S$ be an orbifold with hyperbolic signature $\sigma$; consider the set of quasiconformal homeomorphisms (deformations) $f: S \rightarrow S^{\prime}$, where $S^{\prime}$ is another orbifold with the same signature than $S$, and such that the ramification values of $x$ and $f(x)$ are the same for all $x \in S$. Two such mappings $f: S \rightarrow S^{\prime}$ and $g: S \rightarrow S^{\prime \prime}$, are equivalent if there exists a biholomorphic function $\phi: S^{\prime} \rightarrow S^{\prime \prime}$ (respecting the ramification values) such that $g^{-1} \circ \phi \circ f$ is homotopic to the identity on $S$ (by a homotopy that fixes the points $x_{j}$ ). The set of equivalence classes of deformations of $S$ is the Teichmüller space of $S, T(S)$. We have that if $S \cong \Delta / \Gamma$, with $\Gamma, \Delta$ as in theorem 2 , or $S \cong H / F$ with $F$ Fuchsian, then $T(S) \cong T(\Gamma)$ and $T(S) \cong T(F, H)$ (usually denoted by $T(F)$ ); see [7] and [13].

\section{The Bers-Greenberg isomorphism for b-groups.}

In [5] I. Kra gave a proof of the Bers-Greenberg theorem for the case of Fuchsian groups. In this section, we will follow his arguments to provide a proof of the theorem in the case of terminal regular b-groups. See also the remark at the end of the proof.

Proof of theorem 3. Let $S$ be an orbifold with hyperbolic signature $\sigma$, and assume that at least one of the ramification values of $\sigma$ is finite. Let $\mathcal{P}$ be a maximal partition on $S$. By theorem 2 we have that the pair $(S, \mathcal{P})$ can be uniformized by a terminal regular b-group $\Gamma$ acting on its invariant component $\Delta$. Remove from $S$ the points with finite ramification value to obtain a surface with punctures $S_{0}$; applying theorem 2 again, we get a terminal regular b-group $\Gamma_{0}$ uniformizing $\left(S_{0}, \mathcal{P}\right)$ on the invariant component $\Delta_{0}$. Consider 
now the set $\Delta_{\Gamma}=\Delta-\{$ fixed points of elliptic elements of $\Gamma\}$. Since we are assuming that the signature of $S$ contains finite ramification values, we have $\Delta_{\Gamma} \neq \Delta$. We also have $\Delta_{\Gamma} / \Gamma \cong S_{0}$, so there exists a holomorphic covering $h: \Delta_{0} \rightarrow \Delta_{r}$ making the following diagram commutative:

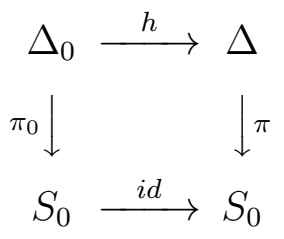

where $\pi_{0}$ and $\pi$ are the natural projections.

$h$ induces a group homomorphism $\chi: \Gamma_{0} \rightarrow \Gamma$ as follows. For any $\gamma \in \Gamma_{0}$, the functions $h$ and $h \circ \gamma$ are lifts of $i d \circ \pi_{0}$ by $\pi$, and therefore there exists an element $g \in \Gamma$ such that $h \circ \gamma=g \circ h$; define $\chi(\gamma)=g$.

Using the map $h$ we can define a norm-preserving isomorphism $h^{*}$ : $L^{\infty}\left(\Gamma_{0}, \Delta_{0}\right) \rightarrow L^{\infty}(\Gamma, \Delta)$, given by $\left(h^{*} \mu\right) \circ h=\mu h^{\prime} / \overline{h^{\prime}}$. One can check (see lemma after the proof) that $h^{*}$ induces a holomorphic mapping between $T\left(\Gamma_{0}\right)$ and $T(\Gamma)$ (which we will also denote by $h^{*}$ ).

$h$ also gives a mapping between quadratic differentials, $h_{*}: Q(\Gamma, \Delta) \rightarrow$ $Q\left(\Gamma_{0}, \Delta_{0}\right)$ by $h_{*} \varphi=\left(\varphi_{0} h\right)\left(h^{\prime}\right)^{2}$. The formula

$$
h^{*}\left(\frac{\overline{h_{*} \varphi}}{\left|h_{*} \varphi\right|}\right)=\frac{\bar{\varphi}}{|\varphi|},
$$

for $\varphi \epsilon Q(\Gamma, \Delta)$, shows that $h^{*}$ takes Teichmüller differentials to Teichmüller differentials and therefore $h^{*}$ is bijective by Teichmüller's theorem.

Remark. One can provide a shorter proof of the above theorem as follows. Given $S$ and $S_{0}$ as above, find Fuchsian groups $F$ and $F_{0}$ such that $H / F \cong$ $S$ and $H / F_{0} \cong S_{0}$. We then have $T(F) \cong T(S) \cong T(\Gamma)$ and $T\left(F_{0}\right) \cong$ $T\left(S_{0}\right) \cong T\left(\Gamma_{0}\right)$. By [4] and [5], we know that $T(F) \cong T\left(F_{0}\right)$, and therefore $T(\Gamma) \cong T\left(\Gamma_{0}\right)$. But for the purpose of this paper we need to have a concrete expression of such an isomorphism, namely $h^{*}$.

Lemma $1 h^{*}: T\left(\Gamma_{0}\right) \rightarrow T(\Gamma)$ is well defined.

Proof. It suffices to consider the case of $\mu$ being equivalent to 0 , i.e. $w \gamma w^{-1}=$ $\gamma$, for all $\gamma \in \Gamma_{0}$, where $w=w^{\mu}$. In that case we get $w\left(\Delta_{0}\right)=\Delta_{0}$ since this 
set is the unique invariant component of $\Gamma_{0}$. Define a mapping $f: \Delta_{\Gamma} \rightarrow \Delta_{\Gamma}$ by $f(h(z))=h(w(z))$. By considering the exact sequence

$$
\{1\} \rightarrow \operatorname{ker} \chi \rightarrow \Gamma_{0} \stackrel{\chi}{\rightarrow} \Gamma \rightarrow\{1\}
$$

it is not hard to see that $f$ is well defined and one-to-one. So we get that $f$ is a quasiconformal mapping whose coefficient is $h^{*}(\mu)$. Extending this coefficient to $\hat{\mathbf{C}}$ by 0 outside $\Delta_{\Gamma}$, we get $f=A \tilde{w}$, where $A$ is a Möbius transformation and $\tilde{w}=w^{h^{*}(\mu)}$.

Let $g \in \Gamma$ and let $\gamma \in \Gamma_{0}$ be such that $\chi(\gamma)=g$. We then have

$$
f g f^{-1} h=f g h w^{-1}=f h \gamma w^{-1}=h w \gamma w^{-1}=h \gamma,
$$

which implies that $\chi(\gamma)=f g f^{-1}$, that is $f g f^{-1}=g$. In particular we have that $A \tilde{w}(x)=x$, for all $x \in \partial \Delta$; since we are assuming that $\infty, 0$ and 1 lie in $\partial \Delta$, we get $A=i d$ and therefore $\tilde{w}$ must be trivial.

\section{Some technical results.}

In this section we will prove some technical lemmas needed in the proof of theorem 1. We deal mainly with three points: first we show that the homomorphism $\chi$ of $\S 2$ takes modular subgroups of $\Gamma_{0}$ onto modular subgroups of $\Gamma$. The second point is a lemma about Teichmüller spaces (deformation lemma) that has interest on its own, and finally we prove some properties of the Maskit embedding.

4.1. Let $S, S_{0}, \Gamma, \Gamma_{0}, \Delta$ and $\Delta_{0}$ be as in $\S 2$. Let $T$ be one of the modular parts of $S_{0}$, and let $\pi^{-1}(T)=\cup_{j \in J} D_{j}$ be a decomposition of the pre-image of $T$ into disjoint connected components (here $\pi$ is the natural projection of diagram 2). Apply $h^{-1}$ and the commutativity of the diagram 2 to get

$$
\pi_{0}^{-1}(T)=h^{-1}\left(\pi^{-1}(T)\right)=\cup_{j \in J} h^{-1}\left(D_{j}\right)
$$

On the other hand, we also have a decomposition into connected components given by $\pi_{0}^{-1}(T)=\cup_{k \in K} A_{k}$. Therefore, for all $k \in K$ there exists a $j \in J$ such that $h\left(A_{k}\right) \subset D_{j}$.

Lemma $2 h\left(A_{k}\right)=D_{j}$. 
Proof. Let us see first that if $A_{k}$ and $A_{l}$ are such that $h\left(A_{k}\right) \subset D_{j}$ and $h\left(A_{l}\right) \subset D_{j}$, then $h\left(A_{k}\right) \cap h\left(A_{l}\right)=\emptyset$ or $h\left(A_{k}\right)=h\left(A_{l}\right)$. So assume $y$ belongs to $h\left(A_{k}\right) \cap h\left(A_{l}\right)$, and let $x_{1} \in A_{k}, x_{2} \in A_{l}$ be such that $h\left(x_{1}\right)=y=h\left(x_{2}\right)$. This gives $\pi_{0}\left(x_{1}\right)=\pi_{0}\left(x_{2}\right)$, so there is a $\gamma \in \Gamma_{0}$ such that $\gamma\left(x_{1}\right)=x_{2}$. In particular we get $\gamma\left(A_{k}\right) \cap A_{l} \neq \emptyset$. Since $\gamma$ is a homeomorphism and $\pi_{0} \circ \gamma=\pi_{0}$, we have $\cup_{p \in K} A_{p}=\cup_{p \in K} \gamma\left(A_{p}\right)$, so $\gamma\left(A_{k}\right)=A_{l}$. We also obtain the equalities

$$
y=h\left(x_{2}\right)=h\left(\gamma\left(x_{1}\right)\right)=\chi(\gamma)\left(h\left(x_{1}\right)\right)=\chi(\gamma)(y) .
$$

Since the only transformation of $\Gamma$ with fixed points in $\Delta_{\Gamma}$ is the identity, we must have $\chi(\gamma)=i d$, and therefore

$$
h\left(A_{l}\right)=h\left(\gamma\left(A_{k}\right)\right)=\chi(\gamma)\left(h\left(A_{k}\right)\right)=h\left(A_{k}\right)
$$

as claimed.

Now to complete the proof of the lemma, let $L$ be the set $L=\{k \in$ $\left.K ; h\left(A_{k}\right) \subset D_{j}\right\}$. Pick any $k_{0} \in L$ and divide $L$ into two disjoint sets, $L=M \cup N$, as follows: $M=\left\{k \in L ; h\left(A_{k}\right)=h\left(A_{k_{0}}\right)\right\}$ and $N=\{k \in$ $\left.L ; h\left(A_{k}\right) \cap h\left(A_{k_{0}}\right)=\emptyset\right\}$. Then $D_{j}=\cup_{k \in L} h\left(A_{k}\right)=\cup_{k \in M} h\left(A_{k}\right) \cup W$, where $W=\cup_{k \in N} h\left(A_{k}\right)$. The sets $h\left(A_{k_{0}}\right)$ and $W$ are open and disjoint, so $W=\emptyset$ (since $D_{j}$ is connected), that is, $N=\emptyset$, giving $h\left(A_{k_{0}}\right)=D_{j}$.

4.2. Choose $D_{j}$ and $A_{k}$ with $h\left(A_{k}\right)=D_{j}$ and rename $A_{j}=A_{k}$ for simplicity. Let $\Gamma_{0}^{j}=\left\{\gamma \in \Gamma_{0} ; \gamma\left(A_{j}\right)=A_{j}\right\}, \Gamma^{j}=\left\{\gamma \in \Gamma ; \gamma\left(D_{j}\right)=D_{j}\right\}$. It is easy to see that $\chi\left(\Gamma_{0}^{j}\right) \subset \Gamma^{j}$, where $\chi$ is the group homomorphism of $\S 2$, but we have a stronger result.

Lemma $3 \chi\left(\Gamma_{0}^{j}\right)=\Gamma^{j}$.

Consider the diagram:

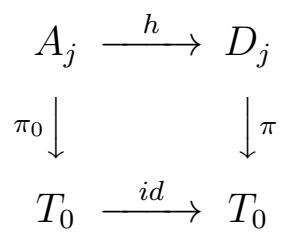

Let $g \in \Gamma^{j}$ and let $z \in D_{j}$. Since $h\left(A_{j}\right)=D_{j}$, there exist points $x, x^{\prime} \in A_{j}$ such that $h(x)=z$ and $h\left(x^{\prime}\right)=g(\gamma)$. We then get $\pi_{0}\left(x^{\prime}\right)=\pi\left(h\left(x^{\prime}\right)\right)=$ $\pi(g(z))=\pi(z)=\pi(h(x))=\pi_{0}\left(x^{\prime}\right)$, so there is $\gamma \in \Gamma^{j}$ such that $\gamma(x)=x^{\prime}$. Now the functions $h \circ \gamma$ and $g \circ h$ satisfy $\pi \circ h \circ \gamma=\pi \circ g \circ h$. So to prove the 
equality $h \circ \gamma=g \circ h$ all we need to do is to check it at one point. Choose $x$ as before; then $h(\gamma(x))=h\left(x^{\prime}\right)=g(z)$ and $g(h(x))=g(z)$, which proves that $\chi(\gamma)=g$ and therefore the lemma.

4.3. Consider now one of the groups we are working with, say $\Gamma$, and a modular subgroup of it, $\Gamma^{j}$. Since $\Delta$ is invariant under $\Gamma$, it will also be invariant under $\Gamma^{j}$, and therefore we can look at the Teichmüller space of $\Gamma^{j}$ supported on $\Delta, T\left(\Gamma^{j}, \Delta\right)=\left\{[\mu] \in T^{j}\right.$; supp $\left.(\mu) \subset \Delta\right\}$, where $[\mu]$ denotes the equivalence class of $\mu$. The following lemma says that this set is actually the whole $T\left(\Gamma^{j}\right)$.

Lemma 4 (Deformation lemma. Analytic version) $T\left(\Gamma^{j}, \Delta\right)=T(\Gamma)$.

Before giving the proof of this lemma, let us look at its geometric meaning. The group $\Gamma^{j}$ is a terminal regular b-group of type $(1,1)$ or $(0,4)$, with invariant component $\Delta^{j}$. The proof is essentially the same in all cases, so we will assume that $\Gamma^{j}$ has signature $(1,1 ; \infty)$, that is, $\Delta^{j} / \Gamma^{j}$ is a torus with one puncture. We have that the quotient $\Delta / \Gamma^{j}$ is a torus with a hole. The difference $\Delta^{j} / \Gamma^{j}-\Delta / \Gamma^{j}$ is a punctured disc $D$. If $\mu \in M\left(\Gamma^{j}, \Delta^{j}\right)$ is supported on $\Delta$, the corresponding homeomorphism induced on $\Delta^{j} / \Gamma^{j}$ will be conformal on $D$ (recall that $\mu=0$ implies $w^{\mu}$ is conformal). Therefore the above lemma is equivalent to the following statement.

Lemma 5 (Deformation lemma. Geometric version.) Let $T$ be a surface with signature $(1,1 ; \infty)$, and let $D$ be a punctured disc on $T$ containing the puncture. Then any quasiconformal deformation of $T$ is equivalent to a deformation that is conformal on $D$.

Proof. Let $f: T \rightarrow T^{\prime}$ be a quasiconformal homeomorphism. Let $D^{\prime}=f(D)$; since $f$ is quasiconformal, $D^{\prime}$ is a also punctured disc. Any two punctured discs are conformally equivalent, so there exists a conformal homeomorphism $g: D \rightarrow D^{\prime}$. Let $h$ denote the function $g \circ f^{-1}: D^{\prime} \rightarrow D^{\prime}$. Let $V^{\prime}$ be a punctured disc on $T^{\prime}$ containing $D^{\prime}$ and such that $V^{\prime}-D^{\prime}$ is annulus. Since $h$ is an orientation preserving homeomorphism, we can extend it smoothly to $T^{\prime}$ so that (the extension of) $h$ is the identity on $T^{\prime}-V^{\prime}$. Now the functions $f$ and $h \circ f$ are homotopic, therefore equivalent, and $h \circ f=g$ is conformal on $D$.

The above proof shows that the result can be generalized to any hyperbolic orbifold as follows. 
Lemma 6 (Deformation Lemma.) Suppose $S$ is an orbifold with hyperbolic signature $\left(p, n ; \nu_{1}, \ldots, \nu_{n}\right)$. Let $D_{1}, \ldots, D_{n}$ be discs around the special points of $S$ (or punctured discs around the punctures) such that their closures are pairwise disjoint. Then any quasiconformal deformation of $S$ is equivalent to a deformation which is conformal on $U_{1} \cup \ldots \cup U_{n}$.

4.4. Our last lemma gives some properties of the Maskit Embedding for Teichmüller spaces of b-groups. As in lemma 4.3., all the proofs are independent (up to technical points) of the ramification values, so for the sake of simplicity we will work out the torsion free situation, that is, the case of b-groups uniformizing compact surfaces with (possibly 0) punctures.

Let us start by looking at the one-dimensional cases, namely those of four times punctured spheres and once punctured tori. A b-group $\Gamma$ of signature $(0,4 ; \infty, \ldots, \infty)$ is constructed by taking two triangle groups, $\Gamma_{1}$ and $\Gamma_{2}$, of signatures $(0,3 ; \infty, \infty, \infty)$, such that $\Gamma_{1} \cap \Gamma_{2}=<A>$, where $A$ is a parabolic element (conjugate in $\operatorname{PSL}(2, \mathbf{C})$ to a translation); then $\Gamma$ is the group generated by $\Gamma_{1}$ and $\Gamma_{2}, \Gamma=\Gamma_{1} *_{<A>} \Gamma_{2}:=<\Gamma_{1}, \Gamma_{2}>$. This is the so-called AFP construction, and the First Combination Theorem [12, Theorem VII.C.2, pg. 149] guarantees that $\Gamma$ is Kleinian if we choose $\Gamma_{1}$ and $\Gamma_{2}$ properly. To fix ideas, let $\Gamma_{1}$ be the group generated by $A(z)=z+2$ and $B(z)=(-z) /(2 z-1)$. Then $\Gamma_{2}=T_{\alpha} \Gamma_{1} T_{\alpha}^{-1}$, where $T_{\alpha}(z)=z+\alpha$, with $\alpha \in \mathbf{C}$. If $\operatorname{Im}(\alpha)$ is big enough, then we can take the horocircle $\left\{z ; \operatorname{Im}(z)=\frac{1}{2} \operatorname{Im}(\alpha)\right\}$ as the invariant curve required in the above quoted theorem, and $\Gamma$ is a bgroup of the desired signature. See figure 1. In [9] (see [2] for the case of torsion. See also [10]) it is proven that $\alpha$ is a global coordinate in the space $T(\Gamma) \cong T(0,4 ; \infty, \ldots, \infty)$. One can write $\alpha$ as the cross ratio $\alpha=$ $\operatorname{cr}(\infty, 0,1, \alpha)$. Observe that the four points involved in this expression are the fixed points of the transformations $A, B, A B$ and $T_{\alpha} B T_{\alpha}^{-1}$, respectively. This way of expressing $\alpha$ as a cross ratio has the advantage of being independent of the particular choice of $\Gamma_{1}$ and $\Gamma_{2}$; i.e., if we start with $D \Gamma_{1} D^{-1}$, for some Möbius transformation $D$, we still have a global coordinate in Teichmüller space expressed as a cross ratio. We can see from the above discussion that the set $\{z ; \operatorname{Im}(z)>k\}$ is contained in $T(\Gamma)$ if $k$ is big emough (actually, $k=1$ works).

For the case of surfaces with signature $(1,1 ; \infty)$, we start with $\Gamma_{1}$ as above, and then we look for a Möbius transformation $C$ such that $C B^{-1} C^{-1}=A$ 
(see [9] for an explanation of why we must take $B^{-1}$ and not $B$ ). This gives $C(z)=\tau+\frac{1}{z}, \tau \in \mathbf{C}$. The horocircle $\left\{z ;\left|z-\frac{i r}{2}\right|=\frac{r}{2}\right\}$ is mapped by $C$ to the horocircle $\left\{z ; \operatorname{Im}(z)=\operatorname{Im}(\tau)-\frac{1}{r}\right\}$. These two curves will be disjoint if $\operatorname{Im}(\tau)>l$, for some positive constant $l$. Then we can apply the Second Combination Theorem [12, VII.E.5, pg. 161] and obtain that the group $\Gamma^{\prime}=\Gamma_{1} *_{C}:=<\Gamma_{1}, C>$ is a b-group with the desired signature. $\tau$ is a global coordinate on $T\left(\Gamma^{\prime}\right)=T(1,1 ; \infty)$, and it can be expressed as the cross ratio $\tau=(\infty, 0,1, C(\infty))$. This construction is called an HNN extension. See figure 2 .

The general b-group $G$ uniformizing a surface with signature $(p, n ; \infty, \ldots, \infty)$ is constructed from triangle groups (actually, $\Gamma_{1}$, its conjugates in $P S L(2, \mathbf{C}$ ) and transformations satisfying relations like $C$ above) by iterated applications of the Combination Theorems. Suppose that we have done the first step and constructed a b-group $G_{1}$ (of type $(0,4)$ or $(1,1)$ ). The following construction will be either an AFP or an HNN extension. In either case, if we use horocircles 'close' to the fixed points of the parabolic elements involved in the process, we are guaranteed that the Maskit Theorems can be applied. But by the cross ratio expression of the coordinates, one sees that these horocirlces correspond to points in Teichmüller spaces with big imaginary parts. So we have proven the following lemma, modulo some technical points that are required for the case of torsion, but they do not present any difficulty.

Lemma 7 For any $\alpha \in T\left(G_{i}\right)$ there exist non-negative real numbers, $y_{\alpha}^{2}, \ldots, y_{\alpha}^{3 p-3+n}$, such that the set $\{\alpha\} \times V_{\alpha}$ is contained in $i(T(G))$, where $V_{\alpha}=\left\{\left(z_{2}, \ldots, z_{3 p-3+n} \in\right.\right.$ $\left.\left.C^{3 p-4+n} ; \operatorname{Im}\right)\left(z_{j}\right)>y_{\alpha}^{j}, j=2, \ldots, 3 p-3+n\right\}$.

Remark. It is clear that this lemma works for any $\alpha_{j}$ in $T\left(G_{j}\right), j=$ $1, \cdots, 3 p-3+n$.

Corollary 1 Given $\alpha$ and $\beta$ in $T\left(G_{1}\right)$, we have $V_{\alpha} \cap V_{\beta} \neq \emptyset$, where $V_{\alpha}$ an $V_{\beta}$ are given by the above lemma.

\section{Proof of theorem 1.}

Let $S, S_{0}, C_{1}, \Gamma, \Gamma_{0}, \Delta$ and $\Delta_{0}$ be as in $\S \S 2,3$. Choose a modular part $T_{j}$ of $S_{0}, 1 \leq j \leq 3 p-3+n$. By lemma 1 we can choose components $D_{j}$ and $A_{j}$ of $\pi^{-1}\left(T_{j}\right)$ and $\pi_{0}^{-1}\left(T_{j}\right)$ respectively, such that $h\left(A_{j}\right)=D_{j}$. By lemma 2 we 
have that $\chi\left(\Gamma_{0}^{j}\right)=\Gamma^{j}$, where $\Gamma_{0}^{j}$ and $\Gamma^{j}$ are defined in 4.2., and $\chi: \Gamma_{0} \rightarrow \Gamma$ is the group homomorphism of $\S 2$. Therefore we can define a function $h_{j}^{*}$ by $\left(h_{j}^{*} \mu\right) \circ h=\mu h_{j}^{\prime} / \overline{h_{j}^{\prime}}$, where $h_{j}$ is the restriction of $h$ to $A_{j}$, and $\mu$ belongs to $T\left(\Gamma_{0}^{j}, \Delta_{0}\right)$. By the deformation lemma we actually have that $h_{j}^{*}$ is defined on $T\left(\Gamma_{0}^{j}\right)$. So we obtain mappings, $h_{j}^{*}: T\left(\Gamma_{0}^{j}\right) \rightarrow T\left(\Gamma^{j}\right), 1 \leq j \leq 3 p-3+n$. Since these mappings are defined by the same differential equation as $h^{*}$, it is clear that the following diagram commutes, if $f$ is equal to the restriction of $\left(h_{1}^{*}, \cdots, h_{3 p-3+n}^{*}\right)$ to $i_{0}\left(T\left(\Gamma_{0}\right)\right)$ :

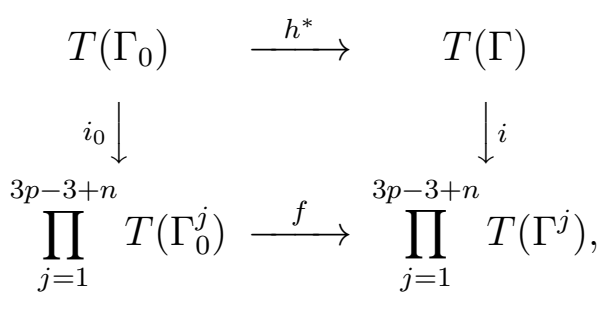

To complete the proof of theorem 1 , all we need to show is that each $h_{j}^{*}$ is bijective. Take $j=1$ to simplify notation.

Injectivity: Let $\alpha, \beta$ be in $T\left(\Gamma_{0}^{I}\right)$ with $h_{1}^{*}(\alpha)=h_{1}^{*}(\beta)$. Lemma 7 gives two open sets, $V_{\alpha}, V_{\beta} \subset C^{3 p-4+n}$ such that $\{\alpha\} \times V_{\alpha}$ and $\{\beta\} \times V_{\beta}$ are contained in $i_{0}\left(T\left(\Gamma_{0}\right)\right)$. By the corollary 1 of the lemma 7 we have $V_{\alpha} \cap V_{\beta} \neq \emptyset$, so we can choose $\gamma$ in that intersection. We then get $f(\alpha, \gamma)=f(\beta, \gamma)$, contradicting the injectivity of $h^{*}$.

Surjectivity: Take $\alpha^{\prime} \in T\left(\Gamma^{1}\right)$, and let $\beta^{\prime}$ be such that $\left(\alpha^{\prime}, \beta^{\prime}\right) \in i(T(\Gamma))$ (which is possible by lemma 6). Then there exists a point $x=(\alpha, \beta)$ in $i\left(T\left(\Gamma_{0}\right)\right) \subset T\left(\Gamma_{0}^{1}\right) \times \prod_{j=2}^{3 p-3+n} T\left(\Gamma_{0}^{j}\right)$ such that $h^{*}(\alpha, \beta)=\left(\alpha^{\prime}, \beta^{\prime}\right)$, giving $h_{1}^{*}(\alpha)=\alpha^{\prime}$.

\section{References}

[1] L. V. Ahlfors, On quasiconformal mappings, J. Analyse Math 3 (19531954), 1-58.

[2] P. Arés, Coordinates for the Teichmüller spaces of b-groups with torsion, Submited to Annal Acad. Sci. Fenn. Ser. A I Math., 1993. 
[3] L. Bers, Quasiconformal mappings and Teichmüller theorem, Analytic Functions (R. Nevanlinna et al., ed.), Princeton Univ. Press, Princeton, NJ., 1960, pp. 53-79.

[4] L. Bers and L. Greenberg, Isomorphisms between Teichmüller spaces, Advances in the Theory of Riemann surfaces, Ann. of Math. Studies 66, 1971, pp. 53-79.

[5] C. Earle and I. Kra, On holomorphic mappings between Teichmüller spaces, Contributions to Analysis, Academic Press, 1974, pp. 107-124.

[6] J. Gentilesco, Automorphisms of the deformation space of a Kleinian group, Trans. Amer. Math. Soc. 248 (1979), 207-220.

[7] I. Kra, On spaces of Kleinian groups, Comment. Math. Helv. 47 (1972), 53-69.

[8] _ Non-variational global coordinates for Teichmüller spaces, Holomorphic functions and Moduli II, Math. Sci. Res. Inst. Publ., vol. 11, Springer, 1988, pp. 221-249.

[9] _ Horocyclic coordinates for Riemann surfaces and moduli spaces. I: Teichmüller and Riemann spaces of Kleinian groups, J. Amer. Math. Soc. 3 (1990), 499-578.

[10] I. Kra and B. Maskit, The deformation space of a Kleinian group, Amer. J. Math. 103 (1981), 1065-1102.

[11] B. Maskit, Moduli of marked Riemann surfaces, Bull. Amer. Math. Soc. 80 (1974), 773-777.

[12] _ Kleinian Groups, Grundlehren der mathematischen Wissenschaften, vol. 287, Springer-Verlag, Berlin, Heidelberg, 1988.

[13] S. Nag, The Complex Analytic Theory of Teichmüller Spaces, John Wiley \& Sons, 1988. 


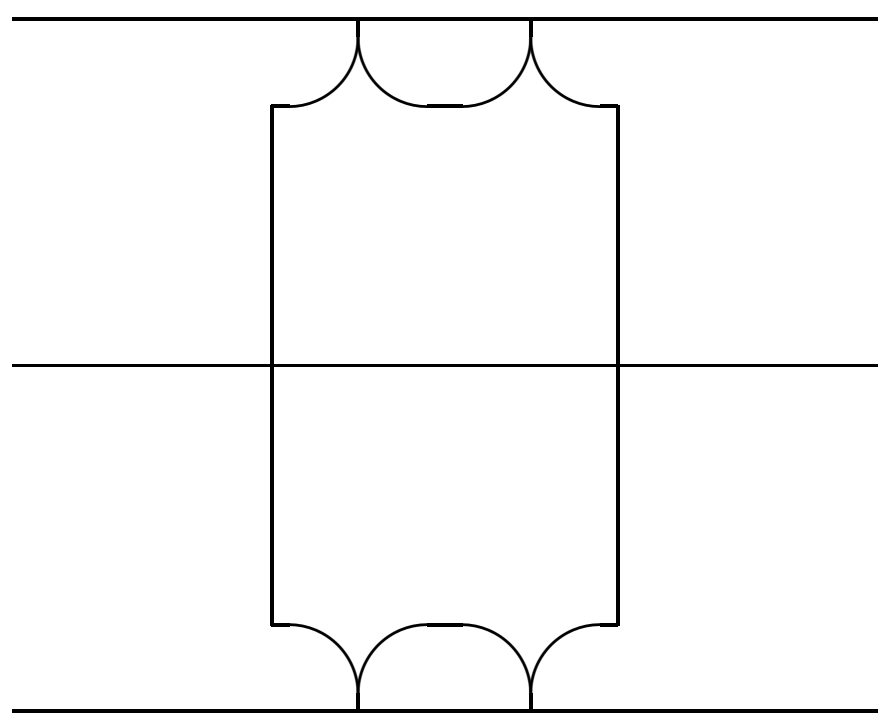

Fig 1. The AFP construction.

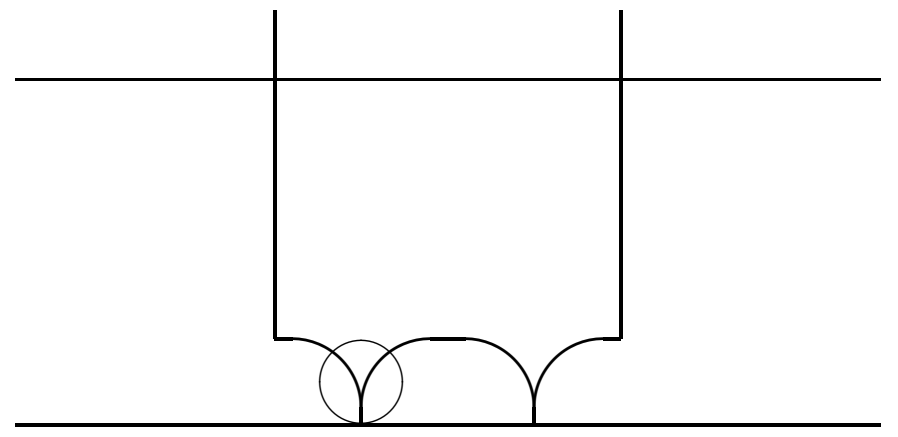

Fig. 2. The HNN extension construction.

Math Department, SUNY at Stony Brook, USA and School of Maths, Tata Institute of Fundamental Research, Bombay, INDIA.

E-mail address: pablo@motive.math.tifr.res.in 